\title{
Estructuras pseudo-comparativas en el latín de Tácito
}

Daniel Gustavo Gutiérrez

Universidad de Buenos Aires, Argentina momolundpolo@gmail.com

Recibido: 13/07/2020. Aceptado: 24/08/2020.

\begin{abstract}
Resumen
El presente trabajo aborda el problema de las estructuras pseudo-comparativas en la lengua latina estandarizada. Problema que supone, por un lado, la caracterización sintáctica, semántica y pragmática de las mencionadas estructuras, y por otro, el análisis centrado en una porción de esa sincronía. A estos fines se ha seleccionado algunas de las obras que constituyen el corpus del historiador y pensador P. Cornelio Tácito. El trabajo parte de las recientes investigaciones y resultados alcanzados por Manfredini (2015).
\end{abstract}

PALABRAS CLAVE: Estructuras pseudo-comparativas, Tácito, problemas de sintaxis latina.

\section{Pseudo-comparative structures in the latin of Tacitus}

\begin{abstract}
The present work addresses the problem of pseudo-comparative structures in the standardized Latin language. Problem that supposes, on the one hand, the syntactic and semantic characterization of the mentioned structures, and on the other, the analysis focused on a portion of that synchrony. For these purposes, some of the works that constitute the corpus of the historian and thinker P. Cornelius Tacitus have been selected. The work builds on the recent research and results achieved by Manfredini (2015).
\end{abstract}

KEYWORDS: Pseudo-comparative structures, Tacitus, Latin syntax problems. 


\section{Introducción}

Considerando el plano del significante (o expresión), puede constatarse en las distintas lenguas la existencia de isomorfías, esto es, de cierta semejanza formal entre dos unidades lingüísticas o gramaticales. ${ }^{1}$ En estos casos, es función del analista indagar en el plano del significado (o contenido) para intentar diferenciar dos (o más) formas isomórficas, orientando el análisis semántico en función de la recategorización de las mismas. En varias lenguas este fenómeno se particulariza, por ejemplo, en la isomorfía manifiesta entre las estructuras que expresan comparación y las estructuras que, aun siendo formalmente semejantes a aquellas, vehiculizan otro tipo de significado. Según Gutiérrez Ordoñez, se denomina estructuras pseudo-comparativas a "aquellos decursos que presentan semejanza formal con las comparativas (pero que no expresan comparación)" (1994:10). El presente trabajo se inscribe en la problemática de la identificación, descripción y especificación sintáctica, semántica y pragmática de las denominadas estructuras pseudo-comparativas en la lengua latina.

A fin de profundizar en esta problemática, se han rastreado ocurrencias de este tipo de construcción en algunas de las obras -o partes de las mismas- del pensador e historiador romano P. Cornelio Tácito (55-c. 120 d.C.), a saber: De vita Iulii Agricolae (c. 96 d.C.), De origine et situ Germanorum (98 d.C.), Dialogus de oratoribus (c. 100 d.C.), Annales 1 (c. 115 d.C.). La elección de dicho corpus supone trabajar con ocurrencias que remiten a una determinada cosmovisión de la sociedad romana así como a ciertas constantes genéricas y estilísticas que responden a las coordenadas histórico-políticas del período imperial que va desde finales del mandato de Domiciano hasta la última etapa del principado de Trajano. ${ }^{2}$

\section{El problema de la pseudo-comparación}

Tanto las expresiones comparativas como las pseudo-comparativas se estructuran en base a dos elementos constitutivos, un adverbio y una conjunción. Por ejemplo, en español:

(i) María más astuta que Ana.

(ii) María astuta más que inteligente.

La diferencia entre ambas expresiones reside en que una estructura comparativa típica (i) señala la diferencia de grado entre dos términos respecto de una propiedad escalar e indica que uno "existe en un grado mayor (superioridad)o menor (inferioridad) que la magnitud, cualidad o acción" (Gutiérrez Ordoñez 1994:53) asociada también al otro término, mientras que una estructura pseudocomparativa típica (ii) implica la adecuación de la asignación de una cierta propiedad a una determinada entidad evaluada como mejor que otra.

Sintácticamente, el adverbio muestra una diferencia posicional en una y otra estructura. Este comportamiento sintáctico parece responder al hecho de que en las expresiones pseudo-comparativas no funcionaría como marcador de grado

\footnotetext{
1 En base a un enfoque de filiación hjelmsleveano, Greimas y Courtés definen el isomorfismo como "la identidad formal de dos o más estructuras (...), reconocibles por la posible homologación entre las redes relacionales que las constituyen" (1990: s.v. isomorfismo).
} 
escalar, sino que se especificaría para focalizar constituyentes. Así, mientras que en una comparación se expresa una confrontación entre estados de cosas, en una pseudo-comparación se advierte una determinada actitud del hablante respecto al dictum, enfatizando que sólo un estado de cosas es válido. ${ }^{3}$

La predicación comparativa pone en juego cuatro elementos: (a) la correlación entre dos entidades $(\mathrm{X} / \mathrm{Y})$, (b) un marcador de grado cuantificacional $(g r)$, (c) un indicador del punto de referencia comparativo $(p r){ }_{,}^{4}(\mathrm{~d})$ una cualidad o propiedad escalar (pe). Si se considera el ejemplo (i), el enunciado puede esquematizarse del siguiente modo:

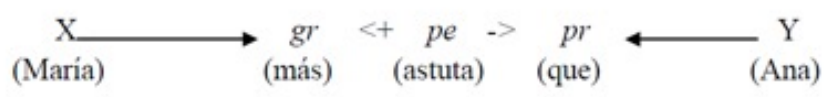

Por su parte, la predicación pseudo-comparativa supone también cuatro elementos constitutivos: (a) la coexistencia de dos propiedades, eventos o situaciones antitéticos (A/no-B), (b) un focalizador no cuantificacional $(f c),{ }^{5}$ (c) el sustrato predicativo sobre el que inhiere la asignación (st), (d) un sujeto percipiente $(s p)$. Se trata, pues, fundamentalmente de un acto subjetivo que involucra un juicio o una valoración: algo (A) es/parece para alguien ( $s p)$ mejor / preferible $(f c)$ que otro algo (no-B), en base a un sustrato predicativo (st). Teniendo en cuenta el ejemplo (ii), lo enunciado quedaría esquematizado de la siguiente manera:

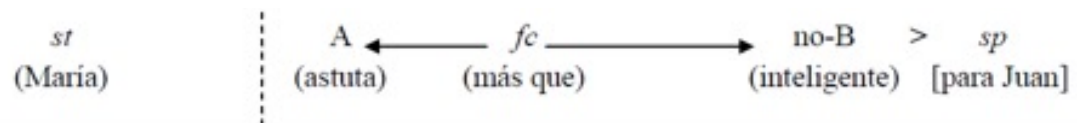

En este tipo de expresión se pone en juego una modalidad epistémica distinta de la que supone una comparación. Mientras que en una comparación se constata la existencia de los hechos, en una pseudo-comparación se hace hincapié en el juicio derivado de la percepción de los hechos. La pseudo-comparación supone, pues, una comparación ya realizada que muestra el compromiso del hablante con lo que está diciendo y / o quiere decir. El siguiente esquema pretende representar todo lo dicho hasta aquí a la vez que sustentar lo que será dicho a continuación:

3 Desde el marco teórico de la filosofía analítica se entiende por 'estado de cosas' una combinación o integración de hechos o entidades: "Der Sachverhalt ist eine Verbindung von Gegenständen (Sachen, Dingen)" (Wittgenstein 1988: prop. 2.01).

4 Gutiérrez Ordoñez señala que las estructuras comparativas "son construcciones que pretenden informar (...) al interlocutor sobre la ubicación de una magnitud a lo largo [de] una escala a partir del punto presuntamente conocido que en dicha escala ocupa otra magnitud (punto de referencia)" (1994:9).

5 En un primer momento la noción de 'focalizador' podría ser pensada en línea con lo que postula Sánchez López acerca de los cuantificadores focales, los cuales "son adverbios que inducen la interpretación cuantitativa del elemento al que modifican por implicación de existencia (o inexistencia) de otros elementos" (1999:1105). Estos cuantificadores "se caracterizan por poder añadir a su significado (...) un matiz valorativo o evaluativo" (1999:1105). Sin embargo, al especificar la semántica de las estructuras pseudo-comparativas (con significado correctivo), Gutiérrez Ordoñez observa puntualmente que "el intensivo más no se aplica a cuantificar y a comparar referentes en una escala de cuantificación: lo que aquí se valora es la propiedad con la que un referente se adscribe a la clase designativa de un signo o a la de otro" (1994:54). En otras palabras, la noción de 'focalizador' es una noción de sesgo pragmático-cognitivo vinculada con la calidad de la información vehiculizada por el sujeto hablante a través de una determinada estructura gramatical, pues, como indica Dik a propósito del concepto de información focal, "in a linguistic expression is that information which is relatively the most important or salient in the given communicative setting" (1997:I 326). Asimismo, según König, "a focus establishes a relation between the value of a focused expression and a set of alternatives" (1991:32). 
Comparación

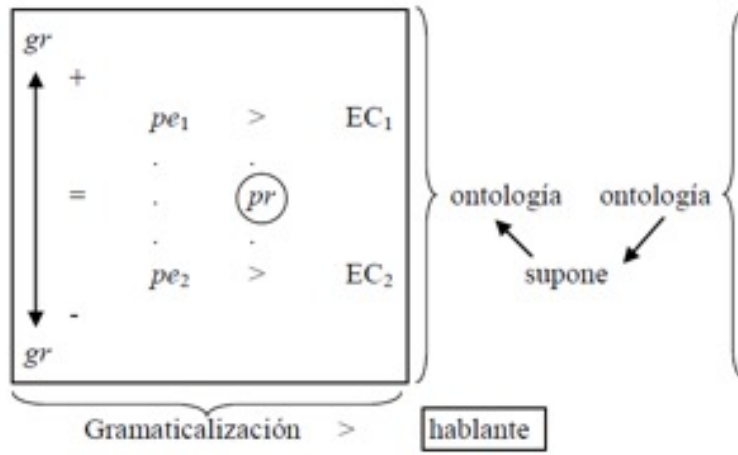

Pseudo-comparación

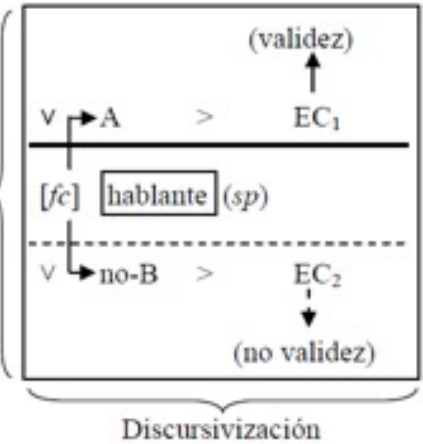

\section{Las estructuras pseudo-comparativas en latín}

En la lengua latina ambas estructuras aparecen también conformadas por los mismos dos elementos: un adverbio y una conjunción (o partícula conjuntiva). El adverbio suele ser preferentemente magis o potius mientras que la conjunción siempre es quam. ${ }^{6}$ A continuación, se propone un ejemplo de expresión comparativa (1) y un ejemplo de expresión pseudo-comparativa (2), ambas con magis:

(1) sed ea sunt consecuta ut magis [mirum sit] accidere illa potuisse [quam nos non vidisse ea futura]. (Cic. Fam. 15.15.2)

Pero sucedió de tal modo que es más admirable que eso haya podido ocurrir que el que nosotros no hubiéramos visto que iba a suceder.

(2) credulitas enim error est magis quam culpa. (Planc. Fam. 10.23.1)

En efecto, la credulidad es un error, más que una culpa. ${ }^{7}$

La fórmula magis... quam parece, según los datos proporcionados por Manfredini (2015), vehiculizar el significado de 'correctividad'. La operación de corrección, que es realizada por el hablante al estructurar la proposición, implica la presencia de un juicio crítico. Mediante esta estructura correctiva el hablante busca otorgarle relevancia a una determinada entidad por sobre otra. Por eso, en tanto expresión de la correctividad, las estructuras pseudo-comparativas con magis... quam conformarían estructuras de foco contrastivo ("A, no B"), a través del cual una determinada entidad lingüística resulta realzada, mientras que otra es rechazada, neutralizada o sustituida. ${ }^{8}$ El hablante, pues, al emitir un juicio crítico, lo hace montado sobre una evidencia. Es por esto que el juicio de corrección involucra tanto una modalidad epistémica (asociada a la certeza y la exactitud de lo que quiere transmitirse: sustituir lógicamente un evento que se revela erróneo, defectuoso o falso por otro acertado, correcto o verdadero) como la manifestación de una evidencia (vinculada con la fuente de la información que sustenta el

6 Cfr. Cabrillana Leal; Tariño Ruiz (2009:651).

7 Si bien estos ejemplos no presentan comparación entre 'propiedades' en relación con individuos (como sí lo hacen los ejemplos en español de la sección anterior), sino de una propiedad en relación con dos eventos (1) o en torno a conceptos (2), téngase en cuenta que estos ejemplos en latín intentan ilustrar el funcionamiento formal de las estructuras, más que detenerse a especificar su contenido lógico-semántico. 
juicio). En las estructuras pseudo-comparativas con magis... quam lo epistémico y lo evidencial suelen aparecer imbricados, ${ }^{9}$ como puede apreciarse en el siguiente ejemplo:

(3) Sed id factum est, non ut vobis rem tam perspicuam dicendo probaremus verum ut omnium malivolorum (...) animos frangeremus (...); tum pecuniam L. Corneli, quae neque invidiosa est et, quantacumque est, eius modi est ut conservata magis quam correpta esse videatur; tum luxuriam, quae non crimine aliquo libidinis, sed communi maledicto notabatur. (Cic. Balb. 56. 2-13)

Pero eso se ha hecho no para probaros con mis palabras un asunto tan claro sino para debilitar el talante de todos los malintencionados (...); por un lado, la riqueza de L. Cornelio, que no es odiosa y, grandiosa como es, es tal que parece ser bien conservada más que rapiñada; por otro lado, (su) suntuosidad, que no era infamada por alguna acusación de deseo desenfrenado, sino por una injuria popular. ${ }^{10}$

Si bien la modalidad epistémica y la modalidad evidencial aparecen frecuentemente superpuestas, es posible, no obstante, diferenciar semánticamente ambas nociones. ${ }^{11}$ Lo epistémico aparece relacionado con el nivel de factualidad (en una gradación que va de la certeza de la ocurrencia a la certeza de la no ocurrencia de un evento) expresada en una proposición, mientras que la evidencialidad mienta las distintas pruebas que el hablante puede proveer para sostener lo que afirma del modo en que lo afirma.

Para Palmer, la diferencia de base entre la modalidad epistémica y la modalidad evidencial consiste en que "with epistemic modality speakers make judgments about the factual status of the propositions, whereas with evidential modality they indicate what is the evidence that they have for it" (2001:24). Cornillie (2009:46) cita a Nuyts, para quien la epistemicidad remite a "the evaluation of the chances that a certain hypothetical state of affairs under consideration will occur, is occurring o has occurred in a possible world" (2001:21), es decir, el juicio evaluativo del hablante se desplazaría a través de un continuum epistémico que va desde el polo de la certeza de la factualidad hasta el polo de la certeza de la no factualidad de lo que dice, pasando por zonas epistémicas intermedias como la probabilidad o la posibilidad (cfr. Cornillie, 2009:46).

Si se concibe este continuum organizado conforme a una suerte de ojiva imaginaria, en el extremo izquierdo se ubicaría la certeza de la factualidad (correlativa gramaticalmente con el modo indicativo, el aspecto perfectivo y la modalidad afirmativa), mientras que en el extremo derecho se ubicaría la zona correspondiente a la certeza de la no factualidad (cuya realización gramatical se condeciría con el modo indicativo y la modalidad negativa); entre ambas se ubicaría una zona intermedia representada por la posibilidad (cuya correlación se da con el empleo del modo subjuntivo y / o el tiempo futuro):

9 Para el solapamiento de lo epistémico y lo evidencial en el ámbito general de la gramática, véase Dik (1997:I 241-243); para el caso específico de la lengua latina, véase Cuzzolin (2010:248-250).

10 Ejemplo sugerido y discutido por la Dra. A. Manfredini en una comunicación personal con el autor de este trabajo, quien se hace responsable de su inclusión aquí ante el juicio del lector. 


\section{El continuum epistémico}

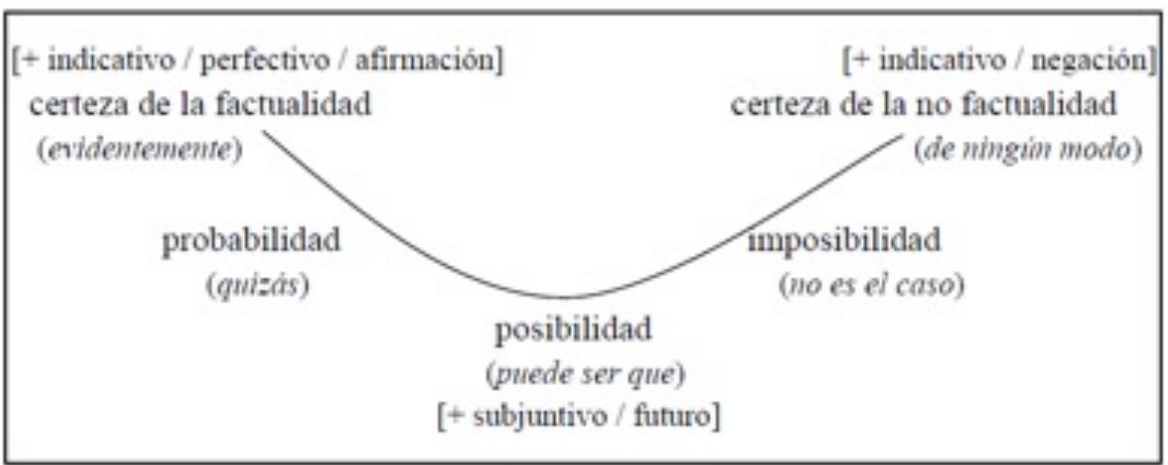

Siguiendo a Aikhenvald (2004), puede decirse que la evidencialidad es una categoría gramatical cuya semántica aparece asociada con la fuente de la información brindada por el hablante así como también con el modo en que esa información es adquirida. La evidencialidad gramatical cubre varios espacios semánticos y cognitivos (realizada frecuentemente en las bases verbales y sus categorías morfológicas: tiempo, modo, aspecto, incluida la categoría no verbal de persona), ${ }^{12}$ a saber: el visual (ver, mirar, etc.), el sensorial (percibir, distinguir, oír, etc.), el inferencial (derivar, inferir, etc.), el presuposicional (suponer, conjeturar, etc.), el dóxico (reporte anónimo, trasmisión indirecta: hearsay; escuchar, enterarse, etc.), el informativo (transmisión directa: quotative; informar, comentar, etc.). ${ }^{13}$

Cornillie define la evidencialidad como una categoría funcional "that refers to the perceptual and/ or epistemological basis for making a speech act" (2009:45). Blakemore (1999) indica que la calidad de la evidencia tiene relación con el grado de certeza que el hablante puede asignar a lo que dice, lo que remite, nuevamente, al solapamiento de ambos aspectos.

Cabe, finalmente, destacar que el latín -así como otras lenguas indoeuropeasno codifica en su sistema gramatical la evidencialidad, ni cuenta con estrategias sistematizadas para expresarla, cosa que dificulta el reconocimiento y análisis de este aspecto vinculado a las estructuras pseudo-comparativas.

Manfredini señala que la fórmula potius... quam es "un poco más versátil que magis quam" (2015:337-338). En efecto, esta fórmula pseudo-comparativa puede vehiculizar un significado correctivo -ejemplo (4)- pero también un significado que lo vincula semánticamente con otra noción, a saber: la de preferencia.

(4) sed mihi ita persuadeo (potest fieri ut fallar) eam rem laudi potius [quam vituperationi] fore. (Cic. Fam. 13.73.2)

Pero estoy así persuadido - puede ser que me equivoque- de que este asunto será para alabanza tuya más bien que para reproche. ${ }^{14}$

\footnotetext{
12 Aikhenvald, por ejemplo, indica que el aspecto perfectivo tiende a indicar conocimiento directo de los hechos (por su valor resultativo), así como el futuro (en tanto mienta eventos no efectivos) se vincula con la inferencia y la especulación $(2004: 109,116)$.
}

13 Cfr. Aikhenvald (2004:23-25), Dik (1997:I 242, 296), Blakemore (1999:141). 
El comportamiento de potius... quam con significado correctivo responde a determinado marco predicativo: (a) presencia de verbos epistémicos y/o manifestación de evidencialidad, (b) contraste focal, (c) expresión de un juicio evaluativo por parte del hablante, (d) semántica basada en la estructura sustitutiva "A, no B". Todo esto lo vincula estrechamente con lo descripto ya para la fórmula magis... quam.

Un marco predicativo diferente es presupuesto por la fórmula con significado de preferencia: (a) uso del modo subjuntivo o el tiempo futuro (implica una modalidad virtual y un matiz volitivo) ${ }_{1}^{15}$ (b) agentividad del sujeto, (c) verbos que poseen el rasgo [+control] (hace al hablante responsable de la ocurrencia del evento):

(5) potius istius culpae crimen (...) suscipiam [quam (...) in te sim crudelis]. (Cic. Ver. 5.105)

Antes asumiré la acusación de esta culpa (...) que ser cruel contigo. ${ }^{16}$

Tarriño Ruiz, siguiendo a Kühner-Stegmann, ${ }^{17}$ indica que las estructuras pseudo-comparativas con potius... quam establecen una relación semántica de exclusión, por la cual entran en relación "cualidades antitéticas o situaciones incompatibles entre sí" (2007:817). La noción de exclusión implica que "la elección o realización de lo uno, marcado por potius, excluye la de lo otro, introducido por quam, de manera que el primer término viene a sustituir al segundo" (2007:817); de este modo "la afirmación de uno de los términos supone la negación del otro" (2007:818). La preferencia, entonces, involucra una alternancia mutua sobre la cual el sujeto elige una alternativa por sobre la otra ("A, antes que B"), lo cual lo lleva a ejercer una acción controlada:

(6) ut longa potius quam lata acies esset. (Liv. 33.8.12)

Para que el frente de combate fuese largo, más bien que ancho. ${ }^{18}$

Otro aspecto que señala la autora es la marcación de una contraexpectativa asociada a esta fórmula pseudo-comparativa, la cual "sucede cuando el hablante quiere alterar información que el oyente ya posee o da por sentada, (...) y en su lugar introduce otra de algún modo inesperada, es decir, que implica una cierta desviación" (2007:818-819):

(7) numquam indicare id filio voluit suo / inopemque optavit potius eum relinquere / quam eum thensaurum commonstraret filio. (Pl. Aul. 11) Nunca quiso enseñárselo a su hijo / y prefirió dejarlo en la pobreza / a mostrarle el tesoro. ${ }^{19}$

Para Tarriño Ruiz, en definitiva, las estructuras pseudo-comparativas con potius... quam responden a la semántica de la exclusión, acarreando implícitamente el elemento descartado, asociado a quam, una negación.

15 Cfr. Baños Baños (2002:56).

16 Ejemplo extraído de Manfredini (2015:347). Cabrillana Leal; Tarriño Ruiz (2009:651) ofrecen el siguiente ejemplo de estructura pseudo-comparativa con significado de preferencia: "dem potius aurum quam illum corrumpi sinam ("le daría el oro antes que permitir que se eche a perder", Plaut. Bacch. 1040)".

17 En Kühner-Stegmann (1914:461) se indica que potius hace recaer todo el peso predicativo en el elemento adyacente, excluyendo de este modo al otro elemento de la estructura.

18 Ejemplo extraído de Tarriño Ruiz (2007:818).

19 Ejemplo extraído de Tarriño Ruiz (2007:819). 
El objetivo del análisis que sigue a continuación consiste en poner a prueba los resultados parciales propuestos por Manfredini (2015), los cuales fueron obtenidos en base a un corpus compuesto, principalmente, por las Epistulae ad Familiares de M. T. Cicerón.

En particular, en la próxima sección de este trabajo se intentará corroborar, proyectar o discutir las apreciaciones de la autora respecto de la diferenciación y especificidad semántica de las estructuras pseudo-comparativas con magis... quam y con potius... quam. ${ }^{20}$ La descripción de las muestras presupondrá todos los aspectos teóricos y metodológicos enunciados hasta aquí.

\section{Análisis del corpus}

Como ya ha sido anunciado al comienzo de este trabajo, el corpus de ocurrencias está conformado por De vita Iulii Agricolae, De origine et situ Germanorum, Dialogus de oratoribus y el libro 1 de Annales, obras todas de P. Cornelio Tácito. ${ }^{21}$ Téngase en cuenta que las obras difieren en género y temática general: una biografía de un ciudadano romano ilustre, un tratado etnográfico sobre un pueblo enemigo de Roma, un diálogo sobre la decadencia de la oratoria en la Roma de finales del siglo I. d.C. y un tratado historiográfico modelado sobre la analística tradicional, respectivamente. Sin embargo, el hilo conductor que cohesiona intencionalmente estas obras se cifra en una precisa y amarga visión crítica de la sociedad romana coetánea al pulso existencial del autor. ${ }^{22}$

La tabla 1 muestra el registro de frecuencia y distribución de las fórmulas magis... quam y potius... quam en el citado corpus:

Tabla 1. Registro de frecuencia y distribución de las fórmulas magis/ potius... quam

\begin{tabular}{|c|c|c|}
\hline magis... quam & potius... quam & Total \\
\hline 17 & 14 & 31 \\
\hline
\end{tabular}

Como puede apreciarse, la frecuencia de aparición de ambas fórmulas es pareja (apenas 3 ocurrencias más para los casos de magis... quam). Este equilibrio exhibe una paridad absoluta al ser discriminados los usos comparativos de los usos pseudo-comparativos, tal y como se muestra en la tabla 2:

Tabla 2. Distribución de usos de las fórmulas magis/potius... quam

\begin{tabular}{|c|c|c|c|}
\hline Uso & Comparativo & Pseudo-comparativo & Total \\
\hline magis... quam & 3 & 14 & 17 \\
\hline potius... quam & 0 & 14 & 14 \\
\hline Total & 3 & 28 & 31 \\
\hline
\end{tabular}

20 La autora dedica también especial atención a las expresiones pseudo-comparativas articuladas con non tam... quam. Por razones metodológicas, estas construcciones (ver el apartado final de este trabajo), así como las articuladas con citius... quam, prius... quam, verius... quam y melius... quam, no serán estudiadas en el marco del presente trabajo, quedando su análisis diferido a una investigación futura.

21 En una primera fase de esta investigación, el corpus estaba conformado por la denominada Opera Minora de Tácito; el número de ocurrencias obligó a ampliar el corpus incluyendo el libro 1 de Annales. 
Por otra parte, se ha diferenciado para los casos de potius... quam las expresiones que portan significado correctivo de las que vehiculizan un significado de preferencia:

Tabla 3. Diferenciación semántica de la fórmula potius... quam

\begin{tabular}{|c|c|c|}
\hline Corrección & Preferencia & Total \\
\hline 9 & 5 & 14 \\
\hline
\end{tabular}

La tabla deja ver que en el corpus seleccionado la frecuencia de uso favorece los casos de potius... quam con significado correctivo. Teniendo en cuenta que los casos de estructuras pseudo-comparativas con magis... quam suelen acarrear de por sí significado correctivo, la diferencia a favor de dicha especificación semántica es contundente:

Tabla 4. Distribución en función de la especificación semántica

\begin{tabular}{|c|c|c|}
\hline $\begin{array}{c}\text { Corrección } \\
\text { (magis/potius... quam) }\end{array}$ & $\begin{array}{c}\text { Preferencia } \\
\text { (potius... quam) }\end{array}$ & Total \\
\hline 23 & 5 & 28 \\
\hline
\end{tabular}

Dado que la noción de correctividad es la que prevalece en el corpus, se comenzará la descripción por las fórmulas que vehiculizan este significado. En primer lugar, serán descritas estructuras con magis... quam (pues son, en definitiva, las que presentan mayor frecuencia de uso con este significado: 14 ocurrencias versus 9 ocurrencias de potius... quam). Luego, serán tratadas las estructuras con potius... quam también con dicho significado, en el intento de postular diferencias en la descripción de ambas fórmulas -en el caso de que las haya. Finalmente, serán descritas las fórmulas con potius... quam que acarrean significado preferencial.

Los ejemplos (8) y (9) ilustran un caso de magis... quam comparativo y un caso pseudo-comparativo, respectivamente:

(8) Faenus agitare et in usuras extendere ignotum; ideoque magis [servatur] quam [si vetitum esset] $]_{\beta}$. (Tac. Ger. 26.1.2) $)^{23}$

Se desconoce perseguir el interés y aumentarlo hasta la usura; y por esto se conserva mejor que si hubiera sido prohibido. ${ }^{24}$

(9) iusserat id M. Ennius castrorum praefectus, [bono $]_{A}$ magis [exemplo $]_{A i}$ quam [concesso iure] $]_{\text {. }}\left(\right.$ Tac. Ann. 1.38.4) ${ }^{25}$

Lo había ordenado Manio Ennio, prefecto del campamento, más por buen ejemplo que por derecho concedido.

En el ejemplo (8) puede apreciarse que al adverbio magis, que aparece antepuesto a "servatur", hace prevalecer su valencia cuantificacional indicando que hay un estado de cosas (conservación saneada de la economía) que se da en grado mayor que otro (posible) estado de cosas (restricción económica) en la idiosincrasia societal de las tribus germánicas. El narrador, al confrontar dos estados de cosas no sólo está constatando la existencia

23 El texto latino de Germania sigue la edición de Anderson (1939).

24 La traducción de todos los pasajes citados pertenece al autor de este trabajo.

25 El texto latino de Anales sigue la edición de Fisher (1906). 
de los hechos sino que informa sobre la ubicación de ellos en una escala de magnitudes. ${ }^{26}$

En el ejemplo (9) puede notarse que magis antecede también al término que focaliza ("exemplo"). Sin embargo, la intención del narrador parece ser bien distinta, pues, aunque supone la existencia de dos acciones (actuar ejemplarmente y obrar conforme a derecho), pondera la asignación de una determinada propiedad perteneciente a un estado de cosas evaluada como mejor que otra. De aquí que quepa preguntarse si lo que aparece focalizado no es "bono" antes que "exemplo"- para ser contrastado con "concesso iure". El contraste, en este caso, opondría dos ámbitos semánticos contrapuestos: el de lo éticocomportamental (actuar ejemplarmente: bonum exemplum) versus el deónticonormativo (obrar conforme a derecho: concessum ius). Téngase en cuenta la situación que se está narrando: Manio Ennio, en calidad de prefecto, ordena ejecutar a dos soldados amotinados. La comparación de (9) con (8) induce dos observaciones: por un lado, manifiesta la actitud del hablante respecto al dictum, enfatizando que sólo una acción debe ser puesta en relieve, por otro, muestra que la evaluación del hablante supone una comparación ya realizada (cuando se trata de hacer valer la autoridad militar en una situación conflictiva resulta más efectivo enseñar el poder mediante el ejemplo contundente que recurrir al derecho establecido).

Otro ejemplo de estructura pseudo-comparativa en la que magis se antepone al término cuyo alcance restringe es el siguiente:

(10) hodie si quid in nobis notitiae ac nominis est, magis arbitror [carminum] A quam [[orationum $]_{B}$ gloria $]_{B i}$ partum. (Tac. Dial. 11.2.6) $)^{27}$

Actualmente, si hay algo en mí de notoriedad y renombre, pienso que ha sido adquirido más por la fama de mis versos que por la de mis discursos.

En términos sintácticos, este ejemplo muestra una mayor semejanza estructural con (8) que con (9), debido a la anteposición de magis respecto del término que marca. No obstante, aparece un elemento que lo diferencia netamente de ambos ejemplos: el verbo arbitror. Este es un verbo que designa procesamiento mental, un verbum intelligendi que, inserto aquí entre el término focalizado ("carminum") por magis y el término introducido por quam ("orationum"), muestra el compromiso cognoscitivo del hablante con lo que está diciendo. El que habla es C. Materno, quien refiere que su reputación literaria está ligada más al cultivo del género poético que al de la oratoria. Como hablante está emitiendo un juicio crítico que intenta otorgarle mayor exactitud a lo que él piensa de sí mismo a la luz de los hechos de los que tiene conocimiento. Al atribuir la causa de su renombre ("gloria") a la dedicación a la poesía, expresa un juicio correctivo, por medio del cual, basado en la percepción que posee de los hechos, se compromete con la validez de lo que afirma.

Algo orientado sutilmente en la misma dirección queda ejemplificado en los ejemplos (11) y (12):

26 A. Manfredini, en una comunicación personal, indica con tino que el segundo hecho no se encuentra constatado (además del problema adicional que supone la comparativa condicional), de modo que en este ejemplo pudiera estar prevaleciendo la dialéctica factual vs. no factual. Que quede enunciado, pues, el carácter complejo del presente ejemplo.

27 El texto latino del Diálogo de los oradores sigue la edición de Furneaux (1939). 
(11) barbari festis epulis, laeto cantu aut truci sonore subiecta vallium ac resultantis saltus complerent, apud Romanos invalidi ignes, interruptae voces, atque ipsi passim adiacerent vallo, oberrarent tentoriis, [insomnes] $]_{A}$ magis [quam pervigiles] . (Tac. Ann. 1.65.5)

Los bárbaros llenaban, en festivos banquetes, con canto alegre o salvaje ruido los estrechos de los valles y de los bosques que resonaban con eco; entre los romanos, débiles fuegos, entrecortadas voces, y estos mismos yacían por todos lados cerca de la empalizada, andaban errantes entre las tiendas, desvelados más que vigilantes.

(12) Caesar (...) vertit ad Rhenum, non auso hoste terga abeuntium lacessere, quod illi moris, quotiens [astu] $]_{A}$ magis [quam per formidinem ${ }_{B}$ ] cessit. (Tac. Ann. 1.56.18)

El César (...) volvió hacia el Rhin, no atreviéndose el enemigo a hostilizar la retaguardia de los que se alejan, lo que es de costumbre para aquel, cada vez que se ha retirado por astucia más que con motivo del temor.

En estos dos ejemplos se hace también patente la actitud del hablante de realzar una determinada entidad gramatical por sobre otra. En efecto, tanto "insomnes" como "astu" operan como términos sustituyentes (del mismo modo que lo hacía "carminum" en el ejemplo anterior), mientras "pervigiles" y el sintagma "per formidinem" lo hacen como términos sustituidos.

Durante la noche, el ejército comandado por Germánico intenta reponerse de la lucha habida durante el día, acción imposibilitada por el estrépito y la algarabía que proviene del campamento de las tribus bárbaras. Es interesante el modo en que es presentado el cuadro por el narrador. Este parece adoptar el punto de vista del observador imparcial que describe una situación. Los datos que posee de las tribus bárbaras son datos de cualidad sonora ("cantu", "sonore", "resultantis"). Esta sería la fuente de información en la que apoya su corrección: voces, ruidos y ecos. Pero en la excitación de la situación bélica, estos datos sensoriales parecerían embotar más que despertar la atención del ejército romano. De ahí entonces que en el ejemplo (11) "insomnes" esté sustituyendo lógicamente un estado de cosas que se revela erróneo o falso por otro considerado acertado o verdadero, siguiendo el esquema de foco contrastivo “A, no B": "insomnes", no "pervigiles".

En (12) también el narrador emite un juicio correctivo: "astu", no "per formidinem", derivado de la percepción de los hechos. En este caso, la evidencia en la que sustenta su juicio proviene de una fuente presuposicional. Germánico asedia el poblado de los catos, tribu germánica cuyos sobrevivientes se dispersan y huyen. Tras la devastación de la aldea, el ejército romano retorna a su enclave en el Rhin sin ser atacado 'por la espalda' por el enemigo derrotado. La intención del narrador en este pasaje consiste en destacar que tal comportamiento no obedece al miedo -natural en el ánimo de los vencidos tras haber experimentado en carne propia una cruel matanza- sino a la astucia de los catos. Tal juicio correctivo se apoya en la 'observación' de las costumbres guerreras de dicho pueblo bárbaro, tal y como es expresado en el inciso que antecede a la estructura pseudo-comparativa ("quod illi moris"). En ambos ejemplos, pues, el narrador activa una operación lógica que responde a una estructura de foco contrastivo puesta en juego por las expresiones pseudocomparativas con magis quam.

Cabe aquí señalar que los ejemplos (11) y (12) muestran que no resulta nada sencillo deslindar lo epistémico de lo evidencial, máxime cuando son 
indicadores de una operación subjetiva del hablante que intenta ofrecer elementos de juicio acerca de la certeza de lo que afirma así como evidenciar la pruebas perceptuales o cognoscitivas que es capaz de proveer para sostener lo que afirma del modo en que lo afirma.

Nótese que en estos dos últimos ejemplos magis y quam aparecen adjuntos. En el corpus la casi totalidad de los ejemplos muestra esta adyacencia entre magis y quam (de las 14 ocurrencias de estructuras pseudo-comparativas tan sólo en 4 aparecen discontinuados). Tal divergencia sintáctica entre los ejemplos podría ser correlativa de una diferenciación semántica.

En (9) se esgrimió la posibilidad de que el elemento focalizado parecía ser más bien el atributo "bono" antes que "exemplo". De ser así, se podría conjeturar que, conforme a los datos brindados por el presente corpus, la adyacencia estaría orientando al interlocutor a percibir una mayor intensidad contrastiva entre los términos relacionados, a la vez que magis quam estaría funcionando en conjunto como un focalizador que opera bidireccionalmente en base a una semántica de la sustitución, activada por un juicio valorativo del hablante montado sobre algún tipo de evidencia. La bidireccionalidad de magis quam se manifiesta en la posposición respecto del término que focaliza (A) y en la anteposición del término que neutraliza (B). La orientación a izquierda del predicado indicaría que se evalúa como más aplicable el primer constituyente. De este modo, la focalización realza tan sólo uno de los constituyentes como el más importante, estableciendo una relación entre el valor que el hablante le otorga a aquello que es por él focalizado y otra entidad que es también por él neutralizada.

Respecto de la modalidad epistémica, se ha visto en (10) cómo aparecía esta manifestada mediante un ítem verbal, mientras que (11) y (12) informaban sobre el sustrato evidencial del juicio epistémico, recurriendo el narrador a ítemes nominales. Hay ejemplos en el corpus que muestran otras posibilidades de expresión de lo epistémico/evidencial:

(13) [argentum] $]_{A}$ quoque magis [quam aurum ${ }_{B}$ ] sequuntur, nulla adfectione animi, sed quia numerus argenteorum facilior usui est promisca ac vilia mercantibus. (Tac. Ger. 5.5.2)

Además, toman por guía la plata más que el oro, no por alguna inclinación de ánimo, sino porque la cantidad de [monedas] de plata es más propicia para el uso que las cosas con las que comprar bienes de intercambio y de poco valor.

El pasaje al que pertenece esta muestra tiene la forma de un diagnóstico de la situación económico-comercial del territorio y del pueblo germano. Un narrador pasa revista del suelo, ganado, mineralogía e intercambio comercial que un grupo de germanos mantiene con la civilización romana. Entre la plata y el oro, este grupo muestra, indica el narrador, una preferencia por el primero de los metales. En este ejemplo, la focalización de "argentum", se ve reforzada por la proposición causal, encabezada por "quia", que sigue después de la construcción pseudo-comparativa. En su estructuración interna, se utiliza el adjetivo "argenteorum" que establece una relación léxico-semántica con el término focalizado por magis. Además, dentro de la proposición causal, el hablante se sirve de una comparación mediante el sufijo -ior. En este ejemplo la proposición causal funciona como una estrategia epistémica / evidencial a la que recurre el hablante con el fin de ofrecer una explicación más precisa de la sustitución de "aurum" por "argentum": la abundancia de la plata contribuye a la practicidad del intercambio comercial, es decir, los germanos valoran el 
uso práctico del metal y no su valor estético-comercial, cosa que sí hacen los romanos. De este contraste de valoraciones y usos emerge, entonces, la estructura de foco contrastivo ("argentum", no "aurum") que utiliza el narrador para emitir un juicio correctivo.

El ejemplo siguiente también apela a la expresión de una causa, sólo que en este caso dicha expresión conforma uno de los constituyentes de la estructura correctiva:

(14) eamque partem Britanniae quae Hiberniam aspicit copiis instruxit, [in spem] $]_{A i}$ magis $\left[\text { quam ob }{ }_{B} \text { formidinem }\right]_{B i}\left(\right.$ Tac. Ag. 24.1.4) ${ }^{28}$

Colocó a las tropas hacia aquella parte de Britania que mira a Hibernia, en vista de una perspectiva [de dominio] más que a causa del miedo.

En el quinto año de la campaña a Britania que conduce Agrícola, busca este consolidar un enclave estratégico que vincule Britania e Hispania. Tras derrotar tribus locales, fortifica hacia el oeste la zona orientada a Hibernia. La preocupación no pasa por la posible hostilidad sino por la posibilidad de robustecer las aspiraciones imperiales de dominio. Por eso, lo sustituido toma la forma de una causa ("ob formidinem") para neutralizarla como tal ("in, no $o b$ "). Lo que persigue el narrador reside en enfatizar la finalidad de la maniobra política llevada a cabo por el individuo cuyas acciones está biografiando y al que además estima como civis romano. La sustitución de "ob formidine" por "in spem" expresa, entonces, la valoración positiva del hablante de un hecho que tuvo lugar en el pasado cercano pero que afecta al futuro del imperio como tal.

Un aspecto que no debería pasarse por alto concierne al papel desempeñado por la información en la articulación de un juicio de corrección:

(15) mox rex vel princeps (...) audiuntur [auctoritate suadendi] $]_{A}$ magis [quam iubendi potestate] $]_{B}$. (Tac. Ger. 11.5.3)

A continuación, el rey o el príncipe (...) son escuchados por la influencia para persuadir más que por el poder para mandar.

En este pasaje el narrador parece esgrimir una valoración positiva de la conducta política de los pueblos germanos en contraposición a lo que acontece en Roma. Entre los bárbaros, aquellos que detentan el poder lo hacen apoyados en una cualidad que la sociedad romana percibe en franca decadencia (amén de las buenas intenciones de Trajano): la posibilidad de dirimir las cuestiones políticas mediante el uso de la palabra ("auctoritate suadendi") y no por el abuso de la violencia ("iubendi potestate"). El contraste entre ambas actitudes de resolución en la esfera de lo público supone una modificación de la información presupuesta, consabida en el año 98. d.C. Apela, pues, al ámbito de lo epistémico-cognoscitivo (creencias, opiniones, saberes) compartido por el hablante y el interlocutor. Era ya proverbial que a partir del principado de Tiberio se había profundizado una de las tendencias más características del mandato de su antecesor: una desmedida inclinación hacia el ejercicio (arbitrario) del poder por parte del princeps, en detrimento del libre funcionamiento de la esfera de lo institucional (simbolizada en el ejercicio de la oratoria y la intervención activa del senado), atribución que le cabría, según la lectura en clave política de la obra de Tácito, tanto a la dinastía Julio-Claudiana como a la dinastía Flavia, término post quem de toda la producción literaria del autor. 
El ejemplo que sigue a continuación ilustra el uso de la fórmula potius... quam con significado correctivo:

(16) quos [Marcus Aper et Iulius Secundus] ego utrosque non modo in iudiciis studiose audiebam, (...), quamvis maligne plerique opinarentur, nec Secundo promptum esse sermonem et Aprum [ingenio] ${ }_{A}$ potius [et [vi naturae $]_{A i}$ [quam [institutione $_{B \mathrm{Bi}}$ et [litteris] $]_{\mathrm{B}}$ famam eloquentiae consecutum. Nam et Secundo purus et pressus et (...), profluens sermo non defuit, et Aper omni eruditione imbutus [contemnebat ${ }_{A}$ ] potius litteras quam [nesciebat ${ }_{B}$ ], tamquam maiorem industriae et laboris gloriam habiturus, si ingenium ${ }_{[a]}$ eius nullis alienarum artium adminiculis inniti videretur. (Tac. Dial. 2.1.12-2.2.4)

Yo los escuchaba con pasión a uno y otro no sólo en los juicios (...), aunque muchos opinaran malintencionadamente que Segundo no tenía un discurso resuelto y que Apro había logrado reputación en la elocuencia por su disposición natural y por el vigor de su carácter más que por la instrucción y la cultura literaria. En verdad, a Segundo no le faltaba un estilo puro, preciso (...), y fluido, y Apro, imbuido de una gran erudición, despreciaba más que desconocía la cultura literaria, [considerando] que habría de obtener de todos modos una gloria mayor por su aplicación y esfuerzo, si parecía que su disposición natural se manifestara sin los puntales de las otras artes.

Afín a lo descripto respecto al par magis... quam como focalizador, en estas estructuras también el término focalizado sustituye, como más aplicable, al que está introducido por quam. En (16) el narrador rememora el encuentro con los oradores Marco Apro y Julio Segundo. Puntualiza en el hecho de la atención que les prestaba cuando estos discurrían acerca del arte retórico. El verbo utilizado ("audiebam"), que mienta una fuente sensorial, remite al tipo de evidencia que parece sobrevolar por todo este comentario. Pues así como escuchaba a los oradores también escuchaba los rumores que sobre estos circulaban ("plerique opinarentur"), saber de oídas asociado a la base evidencial que la bibliografía específica denomina hearsay o reporte anónimo, esto es, un tipo de información que el sujeto posee sin poder especificar su fuente. El rumor está presentado en base a una estructura pseudo-comparativa con la cual se realza lo relativo a la espontaneidad del orador ("ingenio et vi naturae") a la vez que se neutraliza lo que atañe a la formación institucional ("institutione et litteris"). De modo que el rumor tiene la estructura de un juicio valorativo montado sobre un esquema de foco contrastivo. Hay asimismo otros elementos que hacen pensar que, en la valoración del narrador, posee más peso su propia base evidencial (lo que él mismo les escucho decir a los oradores como testigo presencial) que lo que dicen los demás. Se daría algo así como una jerarquización de la base evidencial en la que sustenta su afirmación. En efecto, no cree en el contenido de los rumores (afirma que son dichos "maligne"). Además, inmediatamente se encarga de corregirlos. Por un lado, vindica el estilo de Segundo, vindicación encabezada por el marcador discursivo nam, el cual cubre de un alto grado de certidumbre lo que dice acerca de este orador. Por otro lado, recurre a una estructura correctiva para enmendar los rumores que pesan sobre Apro. Este, afirma el narrador, no ignoraba la cultura literaria sino que la despreciaba; el sentido correctivo es muy preciso: "contemnebat litteras, nesciebat litteras", ${ }^{29}$ lo que responde, por la polaridad modal inherente al segundo verbo, al esquema "A ("contemnebat"),

29 Cabrillana Leal; Tarriño Ruiz (2009:652) dan éste como ejemplo de estructura pseudo-comparativa con significado correctivo: "contemnebat potius litteras quam nesciebat", que traducen "despreciaba la literatura, más que ignorarla". En los casos en que la negación de la predicación está en el lexema verbal (como sucede con "nesciebat litteras", por ejemplo), se niega la afirmación correlativa ("sciebat litteras"). 
no B ("nesciebat")", que se revela antitético con lo que postulaban los rumores. De este modo, el peso de la focalización, ya desde la primera estructura correctiva, parece recaer puntualmente sobre ingenio (funcionando "vi naturae" como una suerte de especificación o suplemento semántico), por lo que resultaría ser el término 'vindicante' que desactiva las falsas acusaciones atribuidas a Marco Apro. Ambas estructuras pseudo-comparativas ponen en juego la sustitución de un término erróneo por otro considerado como más apropiado, correcciones sustentadas sobre un valor epistémico diferente por sujetos diferentes: percepción certera del narrador en el segundo caso, apreciación hipotética de los otros en el primero. El pasaje posee, pues, un sesgo metacrítico, en tanto la antítesis es planteada entre el ingenium, como propiedad característica de Apro en tanto orador, y las litterae. En base a las certezas esgrimidas y desde el punto de vista del narrador, dicha antítesis es resuelta a favor del ingenium de Apro, término positivo e isotópico de este pasaje.

En contraposición con el ejemplo anterior, en el ejemplo (17) el estatus epistémico-evidencial sobre el que se apoya la estructura pseudo-comparativa es comparativamente más lábil, aunque igual de efectivo en la construcción del mensaje:

(17) Sed transeo ad Latinos oratores, in quibus non Menenium, ut puto, Agrippam, qui potest videri antiquus, nostrorum temporum disertis anteponere soletis, sed Ciceronem et Caesarem et Caelium et Calvum et Brutum et Asinium et Messallam: [quos quid [antiquis] ] potius [temporibus] ${ }_{\text {Ai ii }}$ adscribatis [quam nostris ${ }_{B}$ ], non video. (Tac. Dial. 17.1.5)

Pero paso a los oradores latinos, entre los cuales, como pienso, no soléis poner a Agripa, que puede ser considerado antiguo, delante de los [oradores] elocuentes de nuestros tiempos, sino a Cicerón, a César, a Celio, a Calvo, a Bruto, a Asinio y a Mesala, a los que no veo por qué los inscribís en tiempos antiguos más que en los nuestros.

En conversación con Mesala y Materno, Apro discute la noción temporal de antigüedad aplicada a ciertos oradores. Encargándose de la oratoria latina, ordena temporalmente una serie de nombre ilustres (Cicerón, César, etc.). Para confeccionar esta sucesión se apoya en la certeza que tiene del conocimiento de la historia utilizando un verbo de fuerte carga cognoscitiva como puto. Así, la galería de oradores ilustres es ordenada en base al punto de referencia que supone el orador Menenio Agripa, cónsul en el año 250 a.C., mientras que los oradores listados pertenecen todos al siglo I. a.C. A continuación, parece objetarles a sus interlocutores la imprecisión que parecen estos cometer al colocar a los oradores listados entre los oradores antiguos. Apro no puede identificar en qué evidencia basan Mesala y Materno su juicio. El uso de non video en esta situación apuntaría su falta de certeza acerca de la supuesta certeza que exhibe el mentado juicio de sus interlocutores. Ante esta carencia epistémica, recurre a un procedimiento de evaluación. Apro, en el caso de la lista de oradores ilustres, posee la certeza ("ut puto") de la anterioridad temporal de Agripa ("Agrippam, qui potest videri antiquus") respecto de Cicerón, César y sus coetáneos. Este ordenamiento se apoya, entonces, en una comparación patente que hace eje en un orador que sabe con seguridad que es más antiguo que los listados. La estructura correctiva de potius... quam presupone esta comparación, en base a la cual Apro corrige y reordena la adscripción temporal, articulada mediante el contraste antiquis temporibus/nostris temporibus. El presente temporal, en definitiva, de Apro -y sus colegas- habilita la corrección epistémica del juicio ajeno. 
Hasta aquí, los ejemplos (16) y (17) revelan que el comportamiento semántico y pragmático de la fórmula potius... quam con significado correctivo es similar a lo ya descripto para la fórmula magis... quam. Sin embargo, a diferencia de la fórmula magis... quam, las construcciones pseudo-comparativas con potius... quam, además de vehiculizar un significado correctivo, pueden acarrear un significado de preferencia:

(18) officiis et administrationibus potius [ $\underline{n o n}_{A}$ peccaturos $s_{A i}$ praeponere, [quam [damnare] Bii $_{\text {cum peccassent }}$ Bi . (Tac. Ag. 19.3.4) En los cargos y administraciones prefería a los que no cometerían faltas antes que castigarlos cuando las cometieran.

El pasaje versa acerca de las primeras actitudes de Agrícola como gobernador de Britania. Entre ellas, la desestimación del castigo y la valoración del arrepentimiento ante las faltas de sus gobernados. Con este criterio elige a los hombres que le habrán de ayudar en su tarea pública. La preferencia (praeponere), indica el narrador, de Agrícola se decanta a favor de hombres probos para evitar así el castigo en caso de que no lo fueran. En el ejemplo prima una relación semántica de exclusión que supone una alternancia mutua ("non peccaturos" versus "peccassent") en vista de la cual el sujeto elige una alternativa por sobre la otra. Es interesante notar la anteposición tanto de potius como de quam respecto de los términos que introducen, lo cual indicaría una mayor proximidad semántica entre los aspectos contrastados (de hecho, existe aquí identidad de la base léxica). El punto esencial que deja traslucir esta muestra reside en la focalización que parece estar operando potius sobre la negación de la base verbal (non). El quam, por su parte, no niega, sino que excluye una alternativa. Queda claro, pues, que aquí no puede tratarse de una comparación porque "peccassent" no es el término de referencia, sino la opción excluida. Como tal, en esta fórmula el elemento excluido, asociado a quam, acarrea una valoración negativa, sin estar necesariamente negado, pues lo que el sujeto desea poner en relación son dos situaciones incompatibles entre sí: no cometer faltas administrativas versus cometerlas, de lo cual se deriva el criterio del acto gubernativo de Agrícola.

El siguiente es otro ejemplo de potius... quam con significado de preferencia:

(19) opposuerunt abeunti arma, minitantes, ni regrederetur; at ille [moriturum] ${ }_{A}$ potius [quam fidem exueret ${ }_{B}$ ] clamitans, ferrum a latere diripuite elatumque deferebat in pectus, ni proximi prensam dextram vi attinuissent. (Tac. Ann. 1.35.17)

Al que intentaba alejarse le opusieron las armas, amenazándolo si no volvía; pero él, vociferando que moriría antes que faltase a la palabra dada, arrancó de su costado la espada y la dirigía a su elevado pecho, si los que estaban cerca no hubiesen contenido con fuerza la asida diestra.

Lo que permite entrever este ejemplo es el sesgo volitivo presente en todo acto subjetivo de elección entre alternativas. Hay una decisión deliberada del sujeto en el juicio de preferencia. En este pasaje del libro 1 de Anales, se narra cómo Germánico, en medio de un motín de las legiones de Germania, llama al orden. Pero crece el descontento de los soldados. En este clima, el jefe militar intenta abandonar la improvisada asamblea. El pasaje contiene un enorme despliegue de energía representado a través de acciones que implican un elevado grado de voluntad por parte de quienes las realizan. Por un lado, Germánico decidiendo alejarse ("abeunti") para reflexionar y proponer un plan que atenúe el conflicto. Por otro, los soldados levantado sus armas para impedirle el paso 
("opposuerunt arma") y lanzando amenazas ("minitantes"). Luego, Germánico desenvainando la espada ("ferrum a latere diripuit") y autoamenazándose de muerte ("elatumque deferebat in pectus"), lo que deriva en una fuerte reacción de parte de algunos de los presentes que retienen la mano del jefe ("proximi prensam dextram vi attinuissent"). Envuelto en esta situación y con el propósito de mitigar la tensión, Germánico intenta convencer a gritos a los soldados para que lo dejen retirarse. Emite lo que parece ser el resultado de un juicio deliberativo: él preferiría morir ("moriturum") antes que no cumplir su palabra ("fidem exueret"). El elemento focalizado por potius, "moriturum", es la única alternativa posible según la visión de Germánico, pues el término introducido por quam, "fidem exueret", no representa una acción que éste pudiera llevar a cabo voluntariamente. A esto se podría deber el que, en el plano del enunciado, la estructura pseudo-comparativa aparezca 'asediada' por tantos verbos que representan acciones controladas ("opposuerunt", "diripuit", "deferebat", "attinuissent", "minitantes"), no resultando llamativo que la única acción no controlada sea aquella por la que el hablante muestra la preferencia ("moriturum"). Nótese que la semántica del verbo morior implica inherentemente un sujeto no agentivo, mientras que la de exuo un sujeto agentivo. La incompatibilidad entre las alternativas está signada por la voluntad de obrar en un cierto sentido ("moriturum") y por la imposibilidad de hacerlo en otro ("fidem exueret"). Así, lo mentado en el plano semántico es invertido por el sujeto en el plano del discurso: la acción agentiva ("exueret") se revela aquí voluntariamente imposible, mientras que la acción no agentiva pudiera tornarse efectiva si el sujeto así lo quisiera. Al servirse de una estructura pseudo-comparativa de preferencia, el narrador da a entender que Germánico, en la situación en la que se encuentra, sólo involuntariamente faltaría a su palabra, mientras que voluntariamente podría darse muerte (como intenta hacerlo inmediatamente después).

El narrador pone en juego en este punto una contraexpectativa. La expectativa en el interlocutor consiste en dar por sentado que morir no es una acción voluntaria mientras que la disociación entre lo dicho y lo hecho sí lo es. El narrador modifica esta información que el interlocutor no tiene razones para poner en duda, desviándola para transmitir un mensaje más preciso y relevante. Con esta estrategia, el sujeto, al mentar ante su auditorio la realización -como posible- de una de las acciones con la exclusión taxativa de la otra en base al esquema "A, antes que B": "moriturum", antes que "fidem exueret", ejerce en el plano de la enunciación una acción controlada. La reputación como militar de Germánico va, pues, garantida en el eventual cumplimiento de esta alternancia.

Considérese, por último, el siguiente ejemplo:
(20) si [patriam parentes antiqua] mallent [quam dominos et colonias novas]

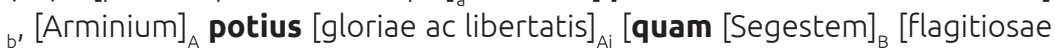 servitutis] $]_{B i}$ ducem] sequerentur. (Tac. Ann. 1.59.21)
Si prefirieran el suelo patrio, sus mayores, sus cosas antiguas que a estos señores y a nuevas colonias, seguirían a Arminio, caudillo de la gloria y la libertad, antes que a Segesto, caudillo de una deshonrosa servidumbre.

Arminio, hostil por causas personales a Tiberio y a Segestes, arenga a los queruscos y a otros pueblos limítrofes contra la política bélica impulsada por el César y su cohorte. El ejemplo es interesante porque expresa dos veces la idea de preferencia: una en la prótasis, otra en la apódosis del período condicional. La primera mediante el verbo malo, cuya semántica implica los rasgos 
[+volición] y [+subjetividad], además de requerir un elemento que complete el esquema de acción que designa. La alternancia electiva se da, aquí, entre los complementos acusativos del verbo ("patriam parentes antiqua") y los términos que introduce quam ("dominos et colonias novas"). La primera alternativa queda planteada entre lo más valioso para los queruscos, esto es, su identidad comunal, y lo que representa la civilización romana: sometimiento material y subjetivo. La otra expresión de la preferencia queda reservada a una estructura pseudo-comparativa con potius... quam. En este caso, la elección parece ser una derivación de la alternancia anterior. El término focalizado por potius, "Arminium", excluye al que es introducido por quam, "Segestem". Porque Segesto representa todo lo negativo: Roma y el sometimiento, mientras que Arminio, lo positivo, lo preferible: la conservación identitaria y la libertad. Ambos términos reciben, además, una aposición especificativa, que parece amplificar y otorgarle peso subjetivo a la preferencia de uno sobre otro. Seguir a Segesto supone someterse a una esclavitud vergonzosa y pasiva ("flagitiosae servitutis"). Estar del lado de Arminio implica el renombre que proviene del no sometimiento ("gloriae ac libertatis"). De esta manera, la alternancia electiva entre el término excluyente ("Arminium") y el término excluido ("Segestem") se fundamenta en la alternancia dicotómica entre libertas y servitus. La presencia de malo en la prótasis es decisiva. Mientras que con la fórmula potius... quam la preferencia se mantiene implícita, la presencia del verbum volendi en el marco predicativo explicita la preferencia como acto de habla.

\section{Conclusiones y proyecciones}

El análisis y las descripciones precedentes permiten alegar que las estructuras pseudo-comparativas son construcciones o estrategias de la lengua latina que aparecen bien diferenciadas en lo semántico y en lo pragmático de las estructuras comparativas.

El examen de la fórmula magis... quam ha revelado lo siguiente: (a) el hablante la utiliza cuando tiene la intención de corregir una atribución errónea, defectuosa o falsa, otorgándole relevancia a una determinada entidad por sobre otra; (b) involucra un juicio crítico montado sobre una evidencia; (c) la modalidad epistémica y lo evidencial no resultan fácilmente deslindables; (d) manifiesta estructuras de contraste focal, en tanto que una entidad es realzada y otra neutralizada o sustituida; (e) responde a la semántica de la sustitución; (f) la disposición sintáctica de magis respecto de quam es correlativa a diferencias semánticas y pragmáticas: la discontinuidad focalizaría más de un constituyente en distintos niveles de saliencia, la adyacencia induciría una focalización bidireccional con mayor intensidad contrastiva.

Por otra parte, la fórmula potius... quam ha mostrado que: (a) el hablante la usa tanto para expresar corrección como para expresar preferencia; (b) el significado correctivo exhibe un comportamiento similar al de la fórmula magis... quam (excepto en el ítem (f), cuya distribución en el corpus no permitió realizar una descripción de este aspecto); (c) cuando el hablante quiere marcar una alternancia sobre la cual emitir un juicio electivo utiliza la fórmula con significado de preferencia; (d) responde a la semántica de la exclusión; (e) es correlativa a la presencia de la subjetividad del hablante y acciones controladas; (f) mientras que potius focaliza una entidad con una elevada carga subjetiva, quam marca o introduce una entidad subjetivamente menos relevante en la consecución del mensaje; (g) algunos factores pragmáticos, por ejemplo, la desviación respecto 
del tipo de información que comparten hablante e interlocutor parece incidir en el uso de esta fórmula. Debido al menor número de ocurrencias, los rasgos aquí listados resultan ser metodológicamente menos precisos que los revelados por la descripción de la fórmula magis... quam, lo cual redunda en una descripción más acotada y de menor proyección explicativa.

La distribución de las fórmulas que se desprende del corpus seleccionado para este trabajo (ver tabla 2) no coincide con lo que sostienen al respecto Cabrillana Leal y Tarriño Ruiz (2009:651), quienes indican que la "forma genérica" para expresar una pseudo-comparación en latín es magis... quam.

Dada la incompletitud de los datos aquí alcanzados y el estado incipiente de este campo de estudio, no resultaría del todo prudente concluir este trabajo con la emisión de un juicio taxativo sobre esta compleja cuestión. Sí sería de provecho hacer intervenir en futuras descripciones variables orientadas a enriquecer el establecimiento de un corpus más abarcativo y sólido que atienda a las especificidades sincrónicas, genéricas, temáticas y estilísticas de las obras de los escritores latinos, así como la introducción de parámetros de naturaleza pragmática y retórico-literaria (como la incidencia del tipo de información y su distribución, el grado de integración entre los elementos vinculados, el peso en la elección de la forma gramatical de la superestructura textual, etc.) a la ya de por sí espinosa problemática que presentan las estructuras pseudocomparativas en latín.

Queda asimismo reservado para una futura investigación indagar otras fórmulas que pudieran integrar estructuras pseudo-comparativas, como non tam... quam, citius... quam, prius... quam, verius... quam y melius... quam. La baja o nula frecuencia de aparición en el corpus de estos binomios pseudo-comparativos tornó dificultosa, cuando no imposible, la tarea de describir su semántica y su funcionamiento textual. Una búsqueda llevada a cabo en PHI en función del corpus establecido arrojó los siguientes resultados: melius... quam (4 ocurrencias), non tam... quam (1 ocurrencia), citius... quam (0 ocurrencias), prius... quam (0 ocurrencias), verius... quam (0 ocurrencias). Sirvan los listados a continuación como ejemplos ilustrativos de un caso de melius... quam comparativo y un caso pseudo-comparativo, respectivamente:

(21) adeo melius est [orationem vel hirta toga induere] ${ }_{a}$ quam [fucatis et meretriciis vestibus insignire $]_{\beta}$. (Tac. Dial. 26.1.4)

Tanto más es mejor vestir el discurso con una toga aun vellosa que adornarlo con prendas teñidas y propias de ramera.

(22) nam statum cuiusque ac securitatem melius [innocentia ${ }_{A}$ ] tuetur quam [eloquentia ${ }_{B}$. (Tac. Dial. 11.4.2)

Pues protege el estado de cosas y la tranquilidad la inocencia más que la elocuencia.

Respecto, entonces, a la adecuación del corpus seleccionado para este trabajo, cabe indicar que se ha mostrado parcialmente productivo para el tratamiento y la descripción de las fórmulas magis... quam y potius... quam, pero que no ha resultado pertinente para el estudio de las otras fórmulas que pueden conformar estructuras pseudo-comparativas en latín, como las mencionadas non tam... quam, citius... quam, prius... quam, verius... quam e incluso melius... quam.

Finalmente, restaría estudiar con detenimiento el problema de las conexiones sintácticas asociadas a este tipo de estructuras, y en especial dentro de la 
lengua latina. Mientras Gutiérrez Ordoñez (1994:55) ve en estas construcciones -restringido al ámbito de la lengua española- un caso de coordinación, Givón -centrando su análisis preferentemente en el inglés- las considera un tipo de subordinación (2001:II 336). Tal indefinición sintáctica parece llevar a Tarriño Ruiz, puntualizando en la problemática específica de la lengua latina, a postular la existencia de una conexión intermedia "entre la coordinación y la subordinación" (2007:823), cuyo eco parece resonar en la "relación conectiva sui generis" de la que habla Manfredini (2015:361). Con el objetivo de clarificar este aspecto sintáctico, no sería improcedente llevar a cabo un tratamiento que involucre parámetros discursivos (semántico-pragmáticos), como, por ejemplo, la medición del grado de coherencia discursiva entre las cláusulas. ${ }^{30}$ Esto último supone trabajar con una metodología que integre lo cualitativo (descripción co-y contextual) y lo cuantitativo (distribución de frecuencias de uso). En el intento por superar la dicotomía coordinación/subordinación, y dada la estructura relacional binaria que presentan las construcciones pseudocomparativas, podría asimismo resultar descriptivamente productivo intentar un análisis sintáctico recurriendo a la noción de interordinación propuesta por Rojo (1978:126). Un problema no menor relativo a esta problemática de las conexiones sintácticas consiste en especificar con claridad conceptual y descriptiva si estas construcciones de la lengua poseen definitivamente $-\mathrm{O}$ no- un estatus clausular.

Como puede apreciarse, la problemática de la pseudo-comparación (en latín y/o en otra(s) lengua(s)) revela que queda aún mucho terreno por explorar. En definitiva, Manfredini (2015:360) califica este campo de estudio de la lengua latina como "un pequeño semillero de dificultades", aserto con el que comulga indefectiblemente el autor del presente trabajo. 


\section{Q Bibliografía}

» Aikhenvald, A. (2004). Evidentiality. Oxford: Oxford University Press.

" Anderson, J. (1939). Cornelii Taciti Opera Minora. Oxford: Oxford University Press.

" Baños Baños, J. M. (2002). "Comparativas con quam y verbo personal en latín". En: Espinilla, E.; Quetglas-Torrego, P. (eds.). La comparación en latín. Barcelona: Universitat de Barcelona; Madrid: Universidad Autónoma de Madrid, 39-62.

" Benario, H. (2012). "The Annals". En: Pagán E. (ed.). A Companion to Tacitus. MaldenOxford: Wiley-Blackwell, 101-122.

» Blakemore, D. (1999). "Evidence and Modality". En: Brown K.; Miller, J. (eds.). Concise Encyclopedia of Grammatical Categories. Amsterdam-Tokyo: Elsevier, 141-145.

" Cabrillana Leal, C.; Tarriño Ruiz, E. (2009). "Finales, consecutivas y comparativas". En: Baños Baños, J. M. (coord.), Sintaxis del latín clásico. Madrid: Liceus, 633-656.

» Cornillie, B. (2009). "Evidentiality and epistemic modality. On the close relationship between two different categories", Functions of Language 16, 44-62.

"Cuzzolin, P. (2010). "Evidentialitätstrategien im Lateinischen. Vorläufige Bemerkungen". En: Anreiter, P.; Kienpointer, M. (eds.). Latin Linguistics Today. Innsbruck: Innsbrucker Beiträge zur Sprachwissenschaft, 243-252.

"Dik, S. (1997). The Theory of Functional Grammar. Part 1: The Structure of the Clause. Berlin-New York: de Gruyter.

» Fisher, C. (1906). Cornelii Taciti Annalium Ab Excessu Divi Augusti Libri. Oxford: Oxford Clarendon Press.

» Furneaux, H. (1939). Cornelii Taciti Opera Minora. Oxford: Oxford University Press.

"Givón, T. (2001). Syntax. An Introduction. 2 vols. Amsterdam-Philadelphia: John Benjamins Publishing Company.

» Greimas, A.; Courtés, J. (1990). Semiótica. Diccionario razonado de la teoría del lenguaje. Madrid: Gredos.

" Gutiérrez Ordoñez, S. (1994). Estructuras pseudocomparativas. Madrid: Arco Libros.

» Kapust, D. (2012). "Tacitus and Political Thought". En: Pagán, E. (ed.). A Companion to Tacitus. Malden-Oxford: Wiley-Blackwell, 504-528.

" König, E. (1991). The Meaning of Focus Particles. A Comparative Perspective. LondonNew York: Routledge.

» Kühner, R.; Stegmann, C. (1914). Ausfürliche Grammatik der lateinischen Sprache. Zweiter Teil. Darmstadt: WBG.

" Manfredini, A. (2015). "Estructuras pseudocomparativas". En: La elipsis verbal en las estructuras comparativas del latín. Zaragoza: Pórtico, 309-363.

"Nuyts, J. (2001). Epistemic Modality, language and conceptualization: A cognitivepragmatic perspective. Amsterdam-Philadelphia: John Benjamins Publishing Company.

" O'Gorman, E. (1993). "No Place like Rome: Identity and Difference in the Germania of Tacitus", Ramus 22, 135-154.

»Palmer, F. (2001). Mood and Modality. Cambridge: Cambridge University Press. 
" Rojo, G. (1978). Cláusulas y oraciones. Santiago de Compostela: Universidad de Santiago de Compostela.

»Rutledge, S. (2012). "Tacitu's Dialogus de Oratoribus". En: Pagán, E. (ed.). A Companion to Tacitus. Malden-Oxford: Wiley-Blackwell, 62-83.

» Sailor, D. (2012). "The Agricola". En: Pagán, E. (ed.). A Companion to Tacitus. MaldenOxford: Wiley-Blackwell, 23-44.

» Sánchez López, C. (1999): "Los cuantificadores". En: Bosque, l.; Demonte, V. (coords.). Gramática descriptiva de la lengua española. Vol. 1. Madrid: Espasa, 1025-1128.

" Tarriño Ruiz, E. (2007). "Pseudo-comparativas en latín: factis potius quam dictis". En: Hinojo, G.; Fernández Corte, J. C. (eds.). Munus quaesitum meritis. Homenaje a Carmen Codoñer. Salamanca: Universidad de Salamanca, 815-824.

» Wittgenstein, L. (1988). Tractatus Logico-Philosophicus. Madrid: Alianza. 